\title{
Yersinia enterocolitica endocarditis on a prosthetic valve
}

\author{
E. Pras, N. Arber, J. Pinkhas and Y. Sidi \\ Department of Internal Medicine D, Beilinson Medical Center, Petah Tikva and the Sackler Faculty of \\ Medicine, Tel Aviv University, Israel
}

\begin{abstract}
Summary: Yersinia entercolitica endocarditis has rarely been described before. This is the first report of prosthetic valve Yersinia enterocolitis endocarditis, complicated by infected brain embolization. The patient, however, completely recovered after 6 weeks of combined therapy with ceftriaxone and gentamicin.
\end{abstract}

\section{Introduction}

Yersinia enterocolitica causes several clinical syndromes. In children the predominant manifestation is acute watery diarrhoea of $3-15$ days duration. In young adults, it may cause right lower quadrant abdominal pain mimicking appendicitis. ${ }^{1}$ Erythema nodosum and arthritis have been implicated with Yersinia infections, the latter in connection with the HLA B-27 histocompatability antigen. ${ }^{2}$ Generalized septicaemia by $Y$. enterocolitica is a condition which usually accompanies chronic diseases, especially liver cirrhosis, and has a poor prognosis. ${ }^{3}$ Only six cases of endocarditis have been previously described. ${ }^{4-9}$ We report a patient with Yersinia endocarditis on a prosthetic valve.

\section{Case report}

A 56 year old male was admitted with left hemiparesis and fever. Three months earlier a prosthetic mitral valve (Starr Edwards M-3) was implanted because of rheumatic heart disease. The operation was successful without fever or any complication in the following 3 months. The patient was regularly treated with frusemide, digoxin and coumarin. Five days prior to admission he began to suffer from fever, shaking chills, vomiting and watery diarrhoea. On the day of admission, a left hemiparesis appeared with deterioration in his mental status.

Examination revealed temperature $39.7^{\circ} \mathrm{C}$, blood pressure $130 / 80 \mathrm{mmHg}$, heart rate $110 / \mathrm{min}$ with atrial fibrillation. Prominent signs of right and left heart failure were found. There was a grade $3 / 6$ systolic murmur over the apex radiating to the left

Correspondence: Y. Sidi, M.D., Department of Medicine D, Beilinson Medical Center, 49100 Petah Tikva, Israel. Accepted: 19 February 1992 axilla. The liver was palpated $3 \mathrm{~cm}$ below the right costal margin and there were multiple splinter haemorrhages. Neurological examination revealed motor and sensory aphasia, and left hemiparesis. The erythrocyte sedimentation rate was 84 /hour, haemoglobin of $12.5 \mathrm{~g} / \mathrm{dl}$, white blood cell count of $13,400 / \mathrm{mm}^{3}$ with a shift to the left. Urinalysis disclosed granular casts. Echo Doppler showed normal movement of the prosthetic mitral valve; no vegetations were demonstrated. The aortic valves was normal. Brain computed tomographic (CT) scan showed a large right parietal infarction. Five out of six blood cultures grew Yersinia enterocolitica (serotype 0.3 , biotype IV, phago-type VIII). Intravenous frusemide, digoxin, ceftriaxone $2 \mathrm{~g}$ and gentamicin $80 \mathrm{mg}$ three times a day, were initiated. After an initial improvement, on the tenth day of hospitalization the patient's mental status deteriorated again. A repeated brain CT showed the development of a brain abscess with a marked mass effect in the region of the infarct. The ceftriaxone dose was doubled to $2 \mathrm{~g}$ twice a day, and mannitol and dexamethasone were added. Gradually the patient's condition improved. Treatment was continued for 6 weeks. Repeated CT scans showed shrinkage of the brain abscess. The patient was discharged to a rehabilitation centre, and after 18 months he is well, with minimal neurological residue.

\section{Discussion}

The frequency of $Y$. enterocolitica infections has greatly increased in the last two decades due to increased awareness of physicians to the clinical syndromes caused by this organism and better techniques of laboratory diagnosis. ${ }^{10}$ Considering the uncomplicated postoperative course, the 3 month gap, and the more recent acute onset of 
diarrhoea and vomiting, we can assume that the infection was not acquired during the postoperative course of the cardiac surgery, but through $Y$. enterocolitica bacteraemia associated with acute gastroenteritis.

There are great differences in the virulence of the different strains of $Y$. enterocolitica. Serotypes 0.3 together with 0.8 and 0.9 are considered to be more virulent than the other types. They occur sporadically and account for most reported cases of bacteremia. ${ }^{11-13}$. In all previous cases of $Y$. enterocolitica endocarditis where serotyping was performed, one of these three serotypes was found..$^{5,6,8}$

Patients with enterocolitis and mesenteric lymphadenitis have a good prognosis, while patients with Yersinia septicaemia have a mortality rate reaching $50 \% .^{14} Y$. enterocolitica sepsis has been commonly reported with predisposing underlying diseases. These include cirrhosis, haemochromatosis, acute iron poisoning, alcoholism, diabetes

\section{References}

1. Vontrappen, G.M., Heg, H.O., Ponnett, E. et al. Yersinia enteritis and enterocolitis, gastroenterological aspects. Gastroenterology 1982, 72: 220-227.

2. Aho, K., Ahvonen, P., Lassus, A., Sievers, K. \& Tiikaimen, A. HL-A 27 in reactive arthritis: a study of yersinia arthritis and Reiter's disease. Arthritis Rheum 1974, 17: 521-526.

3. Spira, T.J. \& Kabins, S.A. Yersinia enterocolitica septicemia with septic arthritis. Arch Intern Med 1976, 136: 1305-1308.

4. Apelbaum, J.S., Wilding, G. \& Morse, L.J. Yersinia enterocolitica endocarditis. Arch Intern Med 1983, 143: 2150-2151.

5. Qunsit, D., Harle, J.R., Fosse, T. et al. Endocardite a yersinia enterocolitica serotype 0:8. Presse Med 1986, $15: 487$.

6. Urbano-Marquez, A., Estrich, R. \& Agusti, A. Infective endocarditis due to yersina enterocolitica. J Infect Dis 1983, 148: 940.

7. Green, H.T., Morris, A.I. \& Haqqani, M.T. Infective endocarditis due to yersinia enterocolitica. $J$ Infect 1983, 3: 267-269.

8. Pedersen, C., Joshepsen, P. \& Hegholm, A. Yersinia enterocolitica endocarditis. Ugeskr-Laeger 1985, 147: 2236-2237.

9. Watanakunakorn, C. Acute infective endocarditis due to yersinia enterocolitica. Am J Med 1989, 86: 723-726.

10. Sryder, J.D., Christenson, E. \& Feldman, R.A. Yersinia enterocolitica infections in Wisconsin. Am J Med 1982, 72: 768-774. mellitus and malnutrition. Interestingly, several of these conditions are associated with iron overload, a known predisposing factor for systemic infection with Y.enterocolitica. ${ }^{15}$ Our patient had none of the diseases mentioned above.

We are aware of only six previous reports of $Y$. enterocolitica endocarditis. Five cases of Yersinia endocarditis have been described on a normal valve, and one on a rheumatic valve..$^{4-9}$ Two of the six previous patients with Yersinia endocarditis have died during the infection. ${ }^{4,5}$ All previous cases, like the present case, presented as acute endocarditis. ${ }^{4-9}$ Abscess formation in the liver, spleen, lung, kidney and heart ${ }^{4,5,12,14,16,17}$ is a common sequence of Yersinia bacteraemia. Abscesses were described in two cases of Yersinia endocarditis in the heart and brain. ${ }^{4,5}$ To our knowledge this is the first description of $Y$. enterocolitica endocarditis on a prosthetic mitral valve.

11. Van Noyen, R., Vandepitte, J., Wauters, G. \& Selderslaghs, R. Yersinia enterocolitica: its isolation by cold enrichment from patients and healthy subjects. J Clin Pathol 1981, 34: $1052-1056$.

12. Bouza, E., Dominguez, A., Meseguer, M. et al. Yersinia enterocolitica septicemia. Am J Clin Pathol 1980, 74: 404-409.

13. Butler, T. Plague and Other Yersinia Infections. Plenum Medical, New York, 1983.

14. Rabson, A.R., Hallett, A.F. \& Koornhof, H.J. Generalized yersinia enterocolitica infection. J Infect Dis 1975, 131: 447-451.

15. Robins-Browne, R.M. \& Prpic, J.K. Effects of iron and desferioxamine on infections with yersinia enterocolitica. Infect Immun 1985, 47: 774-779.

16. Foberg, U., Fryden, A., Kihlstrom, E., Persson, K. \& Weiland, O. Yersinia enterocolitica septicemia: clinical and microbiological aspects. Scand J Infect Dis 1986, 18: 269-279.

17. Saliou-Diallo, G.I., Kim, A., Pike, J. \& Rao, G.M. Fatal yersinia enterocolitica septicemia in a compromised host. $J$ Kans Med Soc 1979, 80: 558-559. 